\title{
La constitution du Conseil Français du Culte Musulman : reconnaissance politique d'un Islam français?
}

\section{Malika Zeghal}

\section{(2) OpenEdition}

Journals

Édition électronique

URL : http://journals.openedition.org/assr/1113

DOI : 10.4000/assr.1113

ISSN : $1777-5825$

Éditeur

Éditions de l'EHESS

Édition imprimée

Date de publication : 1 janvier 2005

Pagination : 97-113

ISBN : 2-7132-2043-2

ISSN : 0335-5985

Référence électronique

Malika Zeghal, «La constitution du Conseil Français du Culte Musulman : reconnaissance politique d'un Islam français? », Archives de sciences sociales des religions [En ligne], 129 | janvier - mars 2005, mis en ligne le 09 janvier 2008, consulté le 30 avril 2019. URL : http://journals.openedition.org/ assr/1113 ; DOI : 10.4000/assr. 1113

Ce document a été généré automatiquement le 30 avril 2019.

(C) Archives de sciences sociales des religions 


\title{
La constitution du Conseil Français du Culte Musulman : reconnaissance politique d'un Islam français?
}

\author{
Malika Zeghal
}

1 En avril 2003, le Conseil Français du Culte Musulman voit le jour, résultat de plus d'une décennie d'efforts, d'hésitations et de débats souvent tendus. La naissance du CFCM est célébrée: impulsée par les pouvoirs publics, elle signale pour nombre d'acteurs, sur le plan à la fois symbolique et institutionnel, la reconnaissance de la place de l'islam, deuxième religion de France. Les musulmans de France se sont dotés d'une «instance représentative » qui, constituée en association régie par la loi de 1901, prendra en charge les questions liées à l'organisation et à la gestion de leur culte. Organisée au niveau national et en conseils régionaux, elle devra « défendre la dignité et les intérêts du culte musulman ; favoriser et organiser le partage d'informations et de services entre les lieux de culte ; encourager le dialogue entre les religions; assurer la représentation des lieux de culte auprès des pouvoirs publics ». On rappelle aussi qu'il s'agit du culte au sens strict, et non de la culture musulmane, « encore moins de la représentation de la communauté musulmane française, pour l'ensemble de ses sujets de préoccupation, qui ne peut se faire que dans le cadre des Institutions de la République ouvertes à tous et donc aux musulmans de France ${ }^{1}$. Mais la création du CFCM est aussi critiquée : le Conseil ferait place trop grande aux courants fondamentalistes, ne serait pas véritablement représentatif de l'ensemble des musulmans français, et surtout, contredirait par l'intervention active de l'État dans sa mise en place, le principe même de laïcité.

2 Constitués, dans le passé, en "cultes reconnus », les catholiques, les protestants et les juifs de France disposaient, avant 1905, d'instances représentatives qui ont évolué dans un rapport souvent tendu avec la République. Aujourd'hui, les instances de ces religions servent d'interlocuteurs avec l'État, dans un contexte où n'existent pas de cultes reconnus au sens juridique. Mais si l'on s'interroge, à travers la création du CFCM, sur la question du mode de « reconnaissance » de l'islam en France, il faut distinguer entre les 
acceptions différentes de ce terme. La procédure mise en place par le ministère de l'Intérieur incite les musulmans de France à s'organiser au sein d'une institution reconnue par celui-ci, au sens où il désire et impulse son établissement, la connaît et l'accepte comme interlocuteur qu'il rend «officiel» par la procédure même: l'État entame ici une relation publicisée - et fortement médiatisée - avec les diverses composantes de l'islam de France qu'il tente de réunir dans une instance unique. Le processus retient la loi de 1905 - qui précise que la République «ne reconnaît pas de cultes » et pose dans le même temps le principe de liberté de culte - comme cadre légal de cette reconnaissance, constamment rappelé aux représentants de l'islam, même si certains acteurs politiques ${ }^{2}$ appellent à sa révision. Les dix années qui mènent à la constitution du CFCM ont aussi soutenu un effort de "connaissance» des diverses composantes de l'islam français de la part des pouvoirs publics qui en font le relevé et entament avec elles un dialogue intense et parfois conflictuel. Relayée par les médias dans la sphère publique, cette mise en avant de l'islam comme religion face à laquelle (et avec laquelle) l'État « devait » s'engager, a fait émerger une sorte de relation privilégiée, passionnée ou apaisée selon les moments, entre la République et l'islam. Cette reconnaissance politique de l'existence d'un Islam français, au sens où les pouvoirs publics prennent acte de sa présence, donne lieu à de nombreuses polémiques sur la place de l'islam en France et plus largement sur les effets de cette reconnaissance politique sur la recomposition des relations entre République et religions. En effet, la procédure de constitution du CFCM a théoriquement pour dessein 1 - de rattraper le décalage historique par rapport à d'autres cultes en soutenant une population musulmane qui dispose de peu de moyens matériels pour organiser ses pratiques collectives et pour qui les discussions avec les pouvoirs publics aux niveaux national et local sont souvent difficiles. La construction de mosquées, la formation et le statut des imams, l'institution de carrés musulmans dans les cimetières, l'organisation et la gestion du marché de la viande hallal, les régulations autour du sacrifice annuel du mouton sont autant de questions de gestion du culte qui peuvent être posées à cette nouvelle instance ; 2 d'identifier et faire émerger un interlocuteur, quitte à créer un «islam officiel », qui une fois installé dans sa fonction de représentation du culte, devra trouver de lui-même sa voie dans les négociations avec les fidèles et les pouvoirs publics. En lui offrant ainsi une visibilité publique, cette sortie de l'islam « des caves et des garages », une expression qui traduit les conditions souvent précaires de l'exercice de l'islam en France, est une forme de reconnaissance politique mais aussi d'intégration d'une partie de la population d'origine musulmane dans une procédure transactionnelle avec les organisations musulmanes ; 3 - pour l'État français mais aussi la société civile, mettre en place le CFCM c'est créer un «Islam français », c'est-à-dire définir et intégrer l'islam dans le cadre de la nation, lui donner des frontières nationales et citoyennes ${ }^{3}$ mais lui permettre aussi de s'exprimer au-delà, en particulier dans le monde musulman, en lui concédant un rôle politique, parfois implicite et instrumentalisé, de médiateur transnational avec l'islam étranger.

3 L'absence doctrinale de clergé en islam rend la création d'une instance représentative de l'islam difficile, mais possible. Des représentants cléricalisés par les pouvoirs politiques ou par l'effet de leur propre charisme jouent le rôle d'autorités religieuses dans le monde musulman; ils souffrent cependant d'une instabilité institutionnelle, une instabilité et une fluidité souvent contenues par des États qui peuvent user d'autoritarisme dans ce domaine. D'où la difficulté : comment faire émerger une instance représentative dans un cadre démocratique comme celui de la France, où il serait difficile d'imposer de manière 
autoritaire une instance qui aurait pour fonction d'être l'interlocutrice des pouvoirs publics sur la question du culte musulman ? Il aura fallu la convergence de plusieurs facteurs pour y parvenir: le lancement d'un processus audacieux en 1999 par J.-P. Chevènement, la volonté active et l'investissement personnel d'un ministre de l'Intérieur, N. Sarkozy, qui a fortement encouragé une partie des organisations musulmanes, parfois de manière autoritaire et politicienne, à organiser l'institution représentative de leur culte sur une base démocratique; la prise de conscience par les leaders des grandes organisations musulmanes en France que leur absence dans une consultation ardemment voulue par les élites au pouvoir ne pourrait que les marginaliser; et les effets du 11 septembre 2001 qui ont poussé les différents protagonistes de cette construction institutionnelle à réguler l'islam à leur avantage et à en maîtriser les manifestations, perçues comme perturbatrices de l'ordre laïque.

Depuis les années 1960, la gestion de l'islam se fait par l'intermédiaire des ambassades des pays du Maghreb, lesquelles négocient avec les pouvoirs publics français les modes de contrôle de leurs coreligionnaires, même s'ils sont français. Par ailleurs, l'islam organisationnel qui, à partir des années 1980, s'est en grande partie constitué sur le mode associatif, s'est construit selon des lignes de fractures nationales et/ou idéologiques. L'islam fondamentaliste et transnational de l'Union des Organisations Islamiques de France (UOIF) proche de l'idéologie des Frères Musulmans se veut intégrationniste sans abandonner son interprétation rigoriste et souvent totalisante de l'islam. La plupart de ceux qui en occupent les postes clés ne sont pas nés en France, sont arabophones et francophones, et se sont installés en France lors de leurs études supérieures ${ }^{4}$. Leur clientèle est jeune, éduquée, multiethnique et française, mais n'est pas forcément en phase avec leur idéologie et peut pratiquer sa religion de manière libre et individualisée ${ }^{5}$. La Fédération Nationale des Musulmans de France, elle aussi rigoriste, est plutôt proche des milieux marocains. Un islam qui se dit plus «laïque », proche de la droite politique française, et lié à l'Algérie depuis 1982, s'est institutionnalisé dans la mosquée de Paris ; celle-ci ne jouit cependant que d'une faible aura, faute d'avoir travaillé son ancrage sur le terrain associatif. D'autres groupes organisés, comme Foi et Pratique, ou les associations représentatives des musulmans turcs font partie de ce paysage éclaté, ainsi que nombre d'associations indépendantes qui font référence à l'islam. Depuis les années 1980 associations et mosquées ont répondu, au niveau local, aux questions du culte, souvent de manière insatisfaisante, incomplète et contradictoire ${ }^{6}$.

5 La prise de conscience par les pouvoirs publics français de la nécessité de reconnaissance publique et politique est tardive. Elle fait suite à la révolution iranienne de 1979, qui les pousse à surveiller de plus près les manifestations de la religion musulmane en France, d'autant que l'islam des années 1980 devient de plus en plus visible dans l'espace public et ajoute aux angoisses politiques que produit la stabilisation migratoire. À partir de 1981, date de l'accession de la gauche au pouvoir, une nouvelle législation permet aux associations régies par la loi de 1901 d'être dirigées par des étrangers, et le nombre de lieux de culte et d'associations islamique croît rapidement. Deux grandes fédérations d'associations naissent à cette époque : l'UOIF, en 1983 puis la FNMF en 1985, des entités dont l'influence est mineure à l'origine, mais qui font concurrence, dans les années 1990, au mouvement des jeunes générations issues de l'immigration, qui encourageait, dans les années 1980, à l'intégration sans passer par la religion. L'UOIF devient influente par le maillage du terrain associatif, comme par ses réseaux transnationaux qui passent par le monde arabo-musulman - en recevant notamment l'aide matérielle et l'influence 
idéologique - diffuse et réadaptée au modèle européen - des États pétroliers du Golfe. La création de nombreux lieux de culte, puis la visibilité des opposants à Salman Rushdie et l'affaire du voile ${ }^{7}$ en 1989 sont autant de signes nouveaux d'un islam qui s'insère et s'exprime activement - sur des modes très diversifiés - dans l'espace public. Ces évolutions inquiètent de nombreux pans de la société civile française, notamment quand il s'agit de l'expression du fondamentalisme ou de groupes radicaux qui recourent à la violence, comme lors des attentats de 1986 ou de 1995 en France ${ }^{8}$. Les médias répercutent ces craintes, sans craindre les amalgames. Ceux-ci se retrouvent dans les représentations qui ne retiennent qu'une partie des jeunes générations musulmanes françaises, celles que la République a échoué à intégrer : les jeunes qui pâtissent particulièrement des effets de la crise économique, qui sont relégués dans les ghettos du chômage et de la délinquance. Ils sont parfois intégrés dans des structures associatives qui font référence à l'islam ${ }^{9}$ et qui peuvent, dans des cas très minoritaires, prendre langue avec l'islam radical transnational. Islam, fondamentalisme ou radicalisme, et jeunes générations issues de l'immigration sont ainsi souvent confondus dans des discours de méconnaissance et d'exclusion symbolique qui redoublent les effets de l'échec de l'intégration sociale, économique et politique d'une partie des seconde et troisième générations issues de l'immigration.

6 Ainsi, à partir de 1989, alors que l'islam a déjà commencé à s'organiser dans des structures locales, nationales et transnationales, constituées «par le bas » et de manière volontaire, les gouvernements successifs initient un processus de construction institutionnelle qui prendra du temps avant d'aboutir. Leurs stratégies manquent de cohésion, face à un islam idéologiquement et structurellement de plus en plus diversifié. Pendant dix ans, les échecs prévalent, en raison du refus des élites politiques d'accepter de prendre en compte toutes les tendances. En 1999, Jean-Pierre Chevènement ${ }^{10}$, alors ministre de l'Intérieur, chargé par sa fonction de gérer les relations entre l'État et les cultes, impulse une nouvelle initiative qui, pour la première fois, entend s'appuyer sur la base la plus large possible, et prend en compte toutes les tendances de l'islam, notamment l'UOIF, que l'intérêt bien compris des politiques incite cette fois à intégrer dans la consultation plutôt que de la marginaliser et la pousser à la radicalisation. C'est un véritable tournant par rapport aux politiques traditionnelles de l'islam des chancelleries. Le 29 octobre 1999, le ministre adresse un courrier aux dirigeants de six fédérations musulmanes, six grandes mosquées et six personnalités musulmanes de France. L'invitation inclura par la suite de nouvelles organisations, attirées par la perspective d'une intégration dans le processus qui prend le nom de "consultation ", reprenant et consacrant le terme arabe d'istichâra. Il s'agit officiellement de faire naître cette instance de représentation du culte musulman dans la transparence et dans la double reconnaissance ${ }^{11}$ : celle de l'islam de France par la République d'une part, celle des lois de la République par les représentants du culte musulman en France d'autre part. Dans un processus paradoxal, les musulmans sont censés s'organiser, mais avec le soutien attentif - parfois transformé en autorité - de l'État. La lettre de J.-P. Chevènement l'énonce de façon explicite: «(...) le temps est passé où l'état pouvait, en une telle matière, dicter sa volonté. Il ne cherchera pas à le faire, en se substituant à la vôtre. Il s'emploiera seulement à vous aider, dans le respect des textes juridiques en vigueur, qui s'imposent à tous. Pourvu que vous souhaitiez vous en saisir, une chance historique peut s'offrir à vous (...). » Les musulmans sont reconnus comme partie de la nation française au sens où l'on rappelle que les textes qui régissent les cultes s'appliquent aussi à l'islam. L'islam est donc, juridiquement, une religion à parité avec les autres : il ne reste plus 
qu'aux musulmans de France d'accepter «l'encouragement » et le soutien logistique du ministère de l'Intérieur pour s'organiser en représentation ${ }^{12}$.

7 La réunion plénière du 28 janvier 2000 au ministère de l'Intérieur définit les critères d'inclusion au processus de la consultation. Les parties y adoptent un texte intitulé «Principes et fondements juridiques régissant les rapports entre les pouvoirs publics et le culte musulman en France » qui précise l'adhésion des membres de la consultation « aux principes fondamentaux de la République française et notamment aux articles 10 et 11 de la Déclaration des droits de l'homme et du citoyen relatifs à la liberté de pensée et à la liberté de religion, à l'article $1^{\text {er }}$ de la Constitution affirmant le caractère laïque de la République et le respect par celle-ci de toutes les croyances, et enfin aux dispositions de la loi du 9 décembre 1905 concernant la séparation des Églises et de l'État ${ }^{13}$. L'acceptation de ce texte est la condition du soutien des pouvoirs publics pour assurer le « rattrapage » par rapport aux autres cultes ${ }^{14}$. Mais la République doit, elle aussi, faire des concessions sur le contenu du texte. Le titre original «Déclaration d'intention relative aux droits et devoirs des fidèles du culte musulman en France », qui suggère de façon un peu trop explicite la suspicion sous-jacente au contrat proposé - reconnaissance politique des membres d'une religion en échange de leur serment de fidélité aux lois de la République française -, est modifié, remplacé par une formulation plus neutre. Quant à la mention sur le droit de changer de religion, elle est supprimée à la demande de l'UOIF. De nombreuses critiques ont été exprimées sur le préalable de la signature de ces principes, mais J.-P. Chevènement parle d'un simple « rappel » des lois de la République, et rattache directement la création du CFCM à la question de la «sécurité de tous les habitants de notre pays ». Il déclare plus loin avec la même ambivalence : « Non que les musulmans en tant que tels puissent être suspects de ne pas se conformer aux lois de la République. Mais il fallait que soit publiquement affirmé qu'il n'existe pas de conflit de principe entre la tradition du culte musulman et l'organisation légale des cultes en France.» Les organisations musulmanes sont ainsi à la fois reconnues et «domestiquées" par la République dans un processus de double reconnaissance politique, qui a pour condition première, pour reprendre l'expression pourtant déniée par le ministre, un «serment de fidélité ${ }^{15}$.

8 L'interprétation de la laïcité française par Jean-Pierre Chevènement, fondée sur le principe de séparation, offre une conception maximale de la laïcité, où la religion ne peut s'épanouir entièrement que dans l'espace privé : « Qu'est ce que la laïcité en effet, sinon la croyance en la capacité de tous les citoyens, quelle que soit leur religion, de s'entendre sur le bien commun, la res publica, à la seule lumière de la raison naturelle à laquelle le Coran se réfère constamment (...). La laïcité préserve, à l'écart de la sphère privée, la sphère publique, un espace où s'épanouit la raison naturelle, que tous les hommes ont en commun (...) » ${ }^{16}$. C'est dans sa version rationaliste que l'islam sera capable de pénétrer et être reconnu dans l'espace public. Cette conception de la laïcité fait clairement apparaître les futures tensions qui naîtront de la volonté d'insérer toutes les sensibilités de l'islam en France dans le processus de consultation, en particulier celle des représentants de l'islam qui interprètent l'islam de manière totalisante.

9 L'arrivée de N. Sarkozy au ministère de l'Intérieur en 2002 marque un tournant dans le processus de consultation qui commençait à battre de l'aile après la signature des "principes et fondements ». Tout autant que Chevènement, le nouveau ministre a l'intention d'œuvrer pour mettre en place le CFCM, mais les méthodes et le discours sont différents. Au niveau de la méthode, le ministre manœuvre pour créer un équilibre viable 
mais qui restera instable, entre des composantes du CFCM qui ont du mal à s'entendre. Il utilise une stratégie qui mêle, selon les besoins, accords bilatéraux - et souvent secrets ou non annoncés - avec les participants mais aussi les ambassades du Maroc et de l'Algérie, reconnaissance personnelle des représentants de l'islam, non exempte de démagogie, et un véritable investissement personnel. On le voit, à grands renforts médiatiques, sillonner la France, aller dans les mosquées, non sans se déchausser avant d'y pénétrer, et rechercher le soutien des imams de mosquées dont certains sont connus pour leur fondamentalisme. Alors que les ministres de l'Intérieur précédents avaient tenté de contenir l'UOIF en privilégiant l'alliance algéro-marocaine, N. Sarkozy travaille, après un début de relations tendues avec l'Union, à raccrocher celle-ci à son grand rival, la mosquée de Paris, dans une alliance utilitaire qui permet de former un consensus temporaire et stratégique, mais peu tenable à moyen terme. L'UOIF est forte du maillage serré qu'elle a patiemment construit sur le terrain associatif alors que la mosquée de Paris, quoique forte du soutien d'Alger, ne détient qu'une faible légitimité sur le terrain. Le 9 décembre 2002, les trois grandes fédérations: mosquée de Paris, FNMF et UOIF signent un protocole d'accord sur la composition du CFCM. Les négociations, d'abord bilatérales puis collectives, mais qui avaient écarté dans leurs premières phases les acteurs dont le poids était considéré comme "mineur» (personnalités et grandes mosquées), culminent avec la réunion à huis clos de l'ensemble des participants (fédérations, grandes mosquées et personnalités) dans le château de Nainville les Roches, une propriété du ministère de l'Intérieur, où le ministre a fait aménager une salle de prières tapissée de moquette verte. L'atmosphère des réunions est tendue, et les participants sont sommés de s'entendre : les personnalités et certaines mosquées, comme les Turcs, ou Foi et pratique se sentent marginalisés, et arguent du fait que la consultation s'est faite à leurs dépens, en favorisant les trois grandes fédérations. La composition du bureau est alors décidée avant même la tenue des élections, et élargie, pour satisfaire l'ensemble des courants : 9 postes pour les fédérations, 5 pour les mosquées, 2 pour les personnalités. Dalil Boubakeur est déjà « nommé » président. Quant à l'UOIF et à la FNMF, elles auront chacune un vice-président pour entourer - ou contenir - le recteur de la mosquée de Paris. Les 6 et 13 avril 2003 les élections des 200 membres de l'assemblée générale et des 41 membres du Conseil d'Administration du CFCM par 4032 grands électeurs désignés par environ 900 lieux de culte ont enfin lieu. Ayant su utiliser les outils du marketing politique, leur présence sur le terrain comme leurs réseaux de clientèle transnationaux, l'UOIF sort gagnante de ce scrutin, forte de 14 sièges au Conseil d'administration et de 8 conseils régionaux sur 22, alors que la mosquée de Paris montre sa faiblesse.

10 Ces négociations bilatérales entre ministère de l'Intérieur et acteurs de la consultation se sont doublées d'un discours volontariste et d'une conception ouverte de la laïcité. Le discours de Nicolas Sarkozy sur la laïcité tranche par rapport à celui de ses prédécesseurs. Comme Chevènement, il s'engage dans une véritable reconnaissance politique de l'islam, mais, plus largement aussi, annonce l'entrée plus active de l'État dans la sphère de la religion en spécifiant que les pouvoirs publics ne sont pas indifférents par rapport aux religions: «(...) la République reconnaît à chacun le droit de vivre sa foi et de la transmettre à ses enfants. La République et la laïcité ce n'est pas la négation de la religion c'est l'égal traitement pour chacune d'entre elles. ${ }^{17}$ » Dans son discours du 29 mars 2003, il déclare : «(...) ce serait une faute pour le ministre chargé des cultes de ne pas partager les grands moments de la vie religieuse, de refuser d'entrer dans une église, dans une synagogue ou dans une mosquée. La religion fait partie de notre société ", évoquant plus 
loin la nécessaire « reconnaissance » de l'islam. Alors que J.-P. Chevènement n'acceptait la présence de la religion dans l'espace public qu'en tant que fondement d'une raison universelle commune, le discours de Sarkozy fait une place plus grande aux « religions » comme croyances, utilisant à plusieurs reprises dans ses discours le terme de « foi », alors que Chevènement favorise notamment l'expression «de tradition musulmane », plus culturelle, et moins émotionnelle. En un sens, Nicolas Sarkozy rend viable la construction d'une instance représentative du culte musulman en élargissant l'espace public à certaines expressions du religieux. Il évoque, en 2004, dans un livre traitant des rapports entre République et religions, qui fait une grande part à la constitution du CFCM, ainsi qu'à sa conception personnelle de la religion, une « laïcité active » opposée à une « laïcité passive ou honteuse ${ }^{18}$. Répondant à la question de savoir si ses perspectives sur le financement public de certaines religions en France pouvaient revenir à « reconnaître tel ou tel culte » : «La réalité, c'est que c'est déjà le cas (...) chacun sait qu'il y a en France de facto des religions "reconnues", qui sont la religion chrétienne, le judaïsme, l'islam, et sur le plan juridique le bouddhisme ${ }^{19}$. Faisant l'équivalence entre "reconnaissance » et acceptation des religions par les pouvoirs publics comme interlocuteurs, l'ex-ministre de l'Intérieur plaide pour une intervention plus active de l'État en matière de religions.

11 Les effets symboliques de la création du CFCM sont extrêmement importants pour les musulmans de France : l'islam est enfin reconnu comme « religion comme les autres » et exclu explicitement de la suspicion réelle ou imaginée. Mais ces effets ne sont pas nécessairement durables et solides, tant ils sont dictés par les hauts et les bas de la politique politicienne, et tant la composition diversifiée du Conseil, forcé de concilier les contraires, tend à fragiliser celui-ci et à le rendre instable à long terme.

En revanche, au-delà des aspects discursifs, on peut noter les effets plus durables de cette reconnaissance. Le processus même de constitution du CFCM donne lieu à la mise en place de «groupes de travail » qui, sur des thèmes particuliers, lancent une réflexion sur l'organisation du culte dans le cadre fixé par la loi de 1905 de séparation et de 1901 sur les associations. Pour le ministère de l'Intérieur, il s'agit de rappeler ce cadre juridique et de communiquer. Les rapports des commissions sur les lieux de culte font ainsi état des avantages offerts par le titre IV de la loi de 1905. Surtout, il y a comparaison avec les pratiques fiscales et associatives des autres cultes ${ }^{20}$ et les problèmes de gestion qu'ils rencontrent également en France ${ }^{21}$. Les commissions font un véritable travail pédagogique: en leur sein, les représentants de diverses sensibilités, assistés des représentants du ministère chargés des questions du culte, posent de nombreuses questions pratiques : mosquées, statut et formation des imams, aumôneries, aïd el-kébir, etc. Sept groupes de travail ont fait l'état des lieux ${ }^{22}$; celui consacré à la question de l'édification des lieux de culte et des carrés musulmans est le plus important et a réuni l'Association des maires de France, la Direction générale des Collectivités locales du ministère de l'Intérieur avec des représentants de la communauté musulmane, confrontant ainsi des acteurs peu habitués à se rencontrer et à échanger sur ces questions difficiles. Les Conseils Régionaux du Culte Musulman fonctionnent aujourd'hui, souvent de manière efficace et moins médiatisée, comme médiateurs entre pouvoirs locaux et communautés musulmanes, à la différence de l'instance nationale du CFCM qui se perd dans des luttes de pouvoir incessantes et avance très lentement dans la résolution des questions liées au culte: le travail de ses 11 commissions commence à la fin de l'année 2003. Certaines sont plus avancées que d'autres, comme la commission pèlerinage qui 
propose la mise en place d'une « mission du pèlerinage » chargée d'assurer la médiation entre les autorités françaises et saoudiennes.

Ainsi la consultation permet-elle la constitution de « relais » et de figures médiatrices qui construisent des relations plus ou moins stables entre les autorités du ministère de l'Intérieur ou les autorités locales et les organisations et mosquées représentées, et peuvent gagner en légitimité auprès de leur propre clientèle comme auprès des pouvoirs publics.

L'action de Nicolas Sarkozy a contribué à transformer la suspicion réciproque entre les gouvernements français et l'UOIF en une fragile reconnaissance réciproque ${ }^{23}$. La cooptation de l'UOIF lui a, certes, fait de la publicité, dans le sens où l'Union a gagné en visibilité ; en contre-partie, celle-ci se trouve maintenant sous le regard du public, donc sous « surveillance » d'une société civile qui n'est pas toujours prête à accepter certains aspects de son idéologie religieuse globalisante. Se présentant comme l'« ami exigeant » des musulmans, Sarkozy a déclaré à la tribune de l'UOIF : « la communauté nationale vous tend la main. Elle vous regarde. Vous êtes désormais comptable de l'image de chaque musulman en France. Saisissez cette main tendue par la République, ne la décevez pas ${ }^{24}$

Ce faisant, le ministre de l'Intérieur, dans une perspective électoraliste, a tenté de gagner des voix auprès de la population musulmane sans forcément s'aliéner ceux qui auraient pu le critiquer pour son rapprochement avec l'UOIF. Ces ambiguïtés montrent que les relations entre le ministère de l'Intérieur et les ténors des associations fondamentalistes n'ont pas fini de fluctuer, à moins que celui-ci (en l'occurrence le/les successeurs de N. Sarkozy à l'Intérieur) ne se désengage à présent des prises de décision et des débats internes au Conseil.

Cependant, si N. Sarkozy introduit pleinement l'UOIF dans le processus de reconnaissance, malgré les critiques, c'est qu'il veut en faire un instrument d'intégration plus large. L'échec de la reconnaissance politique et sociale d'une partie des jeunes générations musulmanes touchée par l'exclusion et la déviance sociale, ou tout simplement de celles que leur identité religieuse affichée marginalise, est explicitement reconnu par N. Sarkozy, qui en cela tranche avec ses prédécesseurs ${ }^{25}$. Cet échec veut être "réglé », pour N. Sarkozy, par la procédure publique de reconnaissance politique, justifiée en partie par le chiffre, très discuté, de " 4 à 5 millions de musulmans ", qui présume de la religion à partir de l'origine ethnique ou nationale ${ }^{26}$. En un sens, la religion peut devenir une instance de régulation du social: "La situation de l'islam de France n'est pas bonne. (...) Une partie de la communauté nationale craint les musulmans et l'islam. Elle s'interroge de façon parfois idéologique sur sa compatibilité avec la République. De l'autre côté, les musulmans se sentent stigmatisés en tant que musulmans par le regard des autres. Or la réalité, c'est que l'islam est là. Il y a 4 à 5 millions de musulmans en France. Le refus de voir cette réalité en face conduit à l'émergence des caves et des garages. Nous avons tout à craindre de cet islam clandestin, car la clandestinité pousse à la radicalisation, alors que l'existence publique pousse à l'intégration et donc à une forme de normalisation ${ }^{27}$. Le CFCM, instrument de reconnaissance, est aussi une institution de domestication, qui rappelle fortement le modèle colonial ${ }^{28}$ de gestion de l'islam.

La conception strictement cultuelle du CFCM s'élargit ainsi, non seulement à la question de la reconnaissance politique, mais aussi à des questions qui concernent la culture musulmane et l'interprétation de l'islam. Étant donné la pluralité des tendances 
interprétatives, issue du mode de désignation du CFCM, il devient inévitable que les questions sur l'organisation du culte se raccrochent à des interrogations interprétatives plus larges. Nicolas Sarkozy, contredisant certaines de ses déclarations, souligne à plusieurs reprises que le débat interne au CFCM devra nécessairement se développer, et appelle ainsi à une conception plus large que celle de simple organisation du culte : « (...) le CFCM sera également un formidable lieu de débats. La question du voile, la place de la femme, autant de questions qui ne sont débattues aujourd'hui ni dans les banlieues ni dans les mosquées. Pour faire émerger un islam de France, on a besoin de ce débat apaisé entre les musulmans français. $"{ }^{29}$ Le ministre dépasse ici la fonction originelle qui est donnée au Conseil, qui est celle d'être l'interlocuteur des pouvoirs publics sur la question du culte et de son organisation. Or, le débat sur les questions d'interprétation risque plutôt de se réaliser en dehors du CFCM, la pluralité de cette institution étant bien plus politique qu'intellectuelle. L'élection du Conseil s'étant faite à partir des lieux de culte, le CFCM est tenu essentiellement par des administrateurs de mosquées et d'associations, qui sont surtout intéressés par les ressources symboliques et la visibilité publique que leur offre leur intégration dans le CFCM. Cela n'empêche pas toutefois les transformations discursives dans le champ religieux de ces grands représentants, évolutions qui passent d'abord par les nécessités politiques, et très peu par des transformations conceptuelles.

Les représentants de l'État ne se privent pas, en revanche, d'intervenir dans les définitions des pratiques et l'interprétation religieuses, se laissant aller à des tentations théologiennes. Par exemple, sur la question de l'abattage du mouton lors de l'aïd el-kébir, qui suscite d'énormes tensions entre les représentants des pouvoirs publics et les musulmans, les autorités locales ou nationales tentent de convaincre, par des arguments théologiques, les musulmans de ne pas effectuer le sacrifice. Le sous-préfet de Mantes-LaJolie rappelle ainsi lors de l'aïd de l'année 2003 à ses co-citoyens musulmans «que la religion musulmane autorise le sacrifice sur trois jours (...) Des pratiques de substitution à l'abattage existent également comme l'obole ou l'accomplissement du sacrifice dans le pays d'origine ». Les représentants concernés par ce texte ont regretté de ne pas être considérés comme Français, et de devoir reconnaitre le sous-préfet comme une autorité religieuse de plus ${ }^{30}$. Recherchant désespérément un interlocuteur religieux qu'il aimerait modeler lui-même mais qui doit rester diversifié en son sein pour respecter les règles d'une société qui se veut pluraliste, et ne pouvant donc le faire émerger de manière autoritaire ou lui faire dire ce qu'il désire, l'État est pris dans ses propres contradictions, et se voit souvent tenté de dépasser le champ étroit de ses prérogatives en matière religieuse.

19 L'insertion de l'UOIF et de la FNMF dans le processus a pour effet de révéler publiquement les divergences et les tensions entre les diverses composantes de l'islam français par des effets de concurrence. Au lendemain des élections du CFCM du printemps 2003 qui consacre la victoire de l'UOIF et de la FNMF et l'échec de la mosquée de Paris, une quinzaine de personnalités signent une pétition qui parait le 12 mai $2003^{31}$. On y trouve Betoule Fekkar-Lambiotte, démissionnaire du CFCM, et très critique d'un processus qui faisait la part belle aux mosquées et fédérations fondamentalistes. Yazid Sabeg, proche de Douste Blazy, qui fonde La Convention laïque des droits pour l'égalité et la promotion des musulmans de France; Tokia Saïfi, secrétaire d'État au développement durable, qui met en place, quant à elle, le Conseil Français des Musulmans Laïques (CFML), dont le congrès fondateur se tient le 24 mai 2003. On peut dessiner ici un parallèle avec le CRIF 
(fédération d'institutions juives à vocation non cultuelle) par rapport au consistoire juif : ces nouvelles créations organisationnelles se veulent plus culturelles et ont des ambitions plus larges que celle, déclarée, de s'occuper du culte au sens «étroit». Mais on peut parier d'emblée que cette deuxième phase institutionnelle fera concurrence - dans l'opposition - au CFCM, ou du moins à certaines de ses composantes. Si ce mouvement recrute dans le sillage des leaders associatifs qui ont émergé lors de la «marche des beurs" de 1983, cette nouvelle institutionnalisation, soutenue explicitement par les pouvoirs publics par l'intermédiaire de Sarkozy et plutôt ancrée à droite ${ }^{32}$, revêt, de manière tout à fait inédite, un habillage religieux. La reconnaissance politique de l'islam a ainsi introduit la variable religieuse dans l'espace de la concurrence politique.

La création d'un islam institutionnel - qui doit être temporairement « officiel »-, produit de l'interaction entre une partie des organisations musulmanes et l'État, par des effets d'inclusion et d'exclusion, fait émerger publiquement les instances marginalisées. La diversité réapparaît au moment même où l'on croit avoir enfin uni les diverses composantes de l'islam dans le CFCM, à l'extérieur de celui-ci. Cet incessant redécoupage institutionnel de l'islam français n'est pas dû à une prétendue incapacité des diverses sensibilités à s'unir - car pourquoi s'uniraient-elles si elles ne peuvent s'entendre sur les interprétations de l'islam qu'elles produisent? Elle est la conséquence directe de la conjonction entre l'absence doctrinale de clergé dans l'islam sunnite et du contexte pluraliste et démocratique dans lequel les divers représentants émergent et s'organisent. La conjonction de ces deux facteurs rend l'accompagnement par l'État du processus d'institutionnalisation de l'islam quasiment impossible par les contradictions qu'il produit, alors que le processus d'organisation du culte a déjà été initié depuis les années 1980 dans le développement des associations et mosquées locales. La proposition que lance D. de Villepin en novembre 2004, qui consiste à créer un Conseil Représentatif des Institutions Musulmanes (CRIM) sur le modèle du Conseil Représentatif des Institutions Juives de France (CRIF), devra faire face à cette difficulté. En effet, le CRIM devrait intégrer d'une part le CFCM, qui se spécialiserait dans la gestion des aspects cultuels, d'autre part des associations musulmanes marginalisées par le processus d'istichâra, qui prendraient en charge une représentation plus culturelle de l'islam, contrebalançant ainsi le poids des représentants fondamentalistes du CFCM. L'État français veut rester le principal soutien de l'institution de représentation du culte musulman: à l'écoute des voix naturellement contradictoires issues des organisations musulmanes françaises, il veut remodeler l'institution pour résoudre cette discordance, renonçant ainsi à se retirer à présent de ce travail d'institutionnalisation pour dialoguer « de l'extérieur » avec les représentants du culte.

21 Il est encore trop tôt, notamment dans la perspective de nouvelles élections des représentants du CFCM en 2005, d'évaluer les changements produits par la mise en place du Conseil au niveau de la gestion du culte. C'est en deçà de l'instance nationale du Conseil, à travers les Conseils régionaux, que les premiers effets semblent être les plus visibles. Les élections de 2003 ont pu confirmer les représentants existants ou en créer de nouveaux, redistribuant les cartes de la représentation et faisant évoluer les rapports qui existaient entre pouvoirs locaux et associations et mosquées. Mais au niveau national, peu de résultats concrets ressortent pour l'instant de cette nouvelle instance qui travaille encore à s'organiser et manque cruellement de moyens financiers: la détermination des dates du mois de Ramadan de l'année 2004 n'a pu se faire, et sur ce point, la constitution du CFCM n'a donc rien changé à la fragmentation que de nombreux fidèles regrettent. 
C'est aussi au-delà du CFCM que d'autres questions sont abordées. Par exemple à travers le juridique, où l'on voit la question du voile sur le lieu de travail se régler face aux prud'hommes, ou à la cour de justice européenne. La politique étrangère permet aussi aux pouvoirs publics de contourner l'existence du CFCM. Sur ce point, la mise en rapport d'une question déjà ancienne en France, celle du port du voile islamique dans les écoles publiques, avec la question récente des otages français en Irak l'illustre parfaitement bien.

La question du port du voile islamique dans les écoles publiques rebondit au lendemain des élections du CFCM, en mai 2003, lancée explicitement par N. Sarkozy lors de sa visite au congrès de l'UOIF du Bourget. Le ministre de l'Intérieur, qui avait suggéré que la question du voile pouvait être discutée par le CFCM, décide paradoxalement de s'en remettre à une autorité religieuse extérieure, à laquelle il rend visite au Caire le 30 décembre 2003 : celle de Mohamed Tantawi, le cheikh d'al-Azhar, la grande institution religieuse sunnite et égyptienne, soumise dans nombre de ses déclarations au régime de Moubarak. Si dans cette démarche, le ministre entend prendre ses distances par rapport au Maghreb, il contourne aussi le CFCM pourtant nouvellement constitué, allant chercher la réponse à ses questions hors de l'espace français, tout en sachant que l'UOIF ne pourra acquiescer à une formule contre le port du voile à l'école publique. La réponse du cheikh d'al-Azhar qui doit contenter ses autorités de tutelle, satisfait superficiellement les demandes de la France, mais de manière ambiguë, un style dans lequel l'institution d'alAzhar a toujours excellé, et qui contredit tout l'esprit du processus de reconnaissance de l'islam entamé par la République française. La fatwâ délivrée par le Grand Imam égyptien ressemble bien à une "dispense", mais elle a des implications profondes sur la représentation de la place des Français musulmans dans la société française. Sa formulation ne met pas en avant l'idée d'un islam français et citoyen et détache la notion de nationalité de celle de citoyenneté. Le Mufti égyptien emploie le mot de "résidence » pour désigner le statut des musulmans de France. Il décrit un islam contraint à se mettre dans une situation de "péché » par la République française, et dessine une opposition radicale entre musulmans et non musulmans au niveau même de la nation française: «(..) si la femme musulmane réside ailleurs que dans un pays musulman (comme la France par exemple), et que les responsables de ce pays décident d'adopter des lois opposées au port du voile, c'est leur droit le plus absolu (...) et je ne peux m'y opposer en tant que musulman. Car ce [les responsables de ce pays] ne sont pas des musulmans. Dans ce contexte précis, lorsqu'une femme musulmane se conforme aux lois d'un pays non musulman, du point de vue de la charia islamique, elle se trouve dans le statut de personne contrainte. » Et, poursuit le Grand Imam, pour conclure son raisonnement, le péché commis sous contrainte ne peut qu'être pardonné par Dieu... L'UOIF ainsi que le Conseil européen de la fatwa souligneront d'ailleurs leur communauté de points de vue sur le voile comme « obligation islamique » avec le Grand Imam et leur déception face à la loi interdisant le port des signes religieux à l'école publique, une loi que le CFCM dans son ensemble avait rejetée, même si la mosquée de Paris s'était à plusieurs reprises prononcée contre le port du voile à l'école. La rentrée 2004, où l'on devait appliquer la nouvelle loi, promettait d'être difficile. Le CFCM et les CRCM s'étaient préparés à jouer un rôle médiateur entre école et familles. Ne se prononçant pas directement sur la question du voile, la production de fatwas ne faisant pas partie de ses prérogatives, le CFCM avait toutefois soutenu " la liberté des jeunes filles à exercer leurs libertés religieuses " ${ }^{33}$. Un événement extérieur, la prise de deux otages français en Irak, où la France n'intervient pas militairement auprès de la coalition dirigée par les États-Unis, et les revendications 
des ravisseurs sur l'annulation de la loi contre le port d'insignes religieux dans les écoles, ont unifié, pour un moment du moins, les différents protagonistes autour des principes de la République laïque. Ces rebondissements ont épargné au CFCM une rentrée difficile sur le plan national et l'ont propulsé dans sa fonction de relais sur la scène internationale à travers ses appels aux ravisseurs, assortis de quelques cafouillages. Mais le temps use cette unité superficielle, et dès la mi-septembre, les dissensions internes au CFCM refont surface, non pas au sujet du voile, provisoirement mis de côté, mais au sujet du mode de scrutin, remis en question une fois de plus par la mosquée de Paris.

Les membres du CFCM ont explicitement reconnu les lois de la République, dans une sorte de "moment concordataire », - car le mot même de « reconnaissance » a été prononcé temporaire et instable, fixé dans une procédure de double reconnaissance politique. Pour nombre d'observateurs, celle-ci déroge au principe de laïcité, mais elle s'avère aussi, pour les pouvoirs publics, être une étape nécessaire. Cette reconnaissance réciproque n'est donc pas acquise une fois pour toutes, puisqu'elle n'est pas inscrite dans une procédure juridique mais dans la conjoncture politique. Elle reste par conséquent fragile, car, en cas d'échec du CFCM elle devra probablement être reconduite. Si, à l'inverse, ce dernier réussit, elle n'aurait, alors, plus lieu d'être. Aujourd'hui encore, les décisions du CFCM se prennent par tractations directes entre le ministère de l'Intérieur qui reste toujours partie prenante, l'UOIF et la mosquée de Paris. Si ces négociations continuent d'être le ressort du fonctionnement du CFCM, le Conseil oscillera probablement entre un « islam quasi-officiel» et un simple organe national de gestion du culte. Mais, au-delà de l'existence et du fonctionnement du CFCM, qui ne peut lui-même saisir la justice, les décisions judiciaires sont de plus en plus influencées par la jurisprudence européenne qui répond souvent positivement aux demandes de reconnaissance des libertés religieuses individuelles. Les droits français et européen peuvent fonctionner comme instruments de "demande de reconnaissance » et peuvent être une manière de contourner le CFCM si celui-ci mécontente certaines aspirations de citoyens français musulmans. Les questions que son existence soulève annoncent toutefois que l'islam est enfin entré dans les débats plus larges sur la place de la religion en France.

\section{NOTES}

1. Discours de Nicolas Sarkozy devant la COMOR le 21 octobre 2002.

2. Nicolas Sarkozy a lui-même, tout en rappelant qu'il tient «à l'esprit de la loi de 1905 », proposé de réaménager celle-ci pour permettre à l'État de financer les religions. Voir Nicolas SARKOZY, La République, les religions, l'espérance, Paris, Cerf, 2004.

3. Voir John BowEN, « Does Islam Have Borders? Dilemnas of Domestication in a Global Religious Field", American Anthropologist, 2004, Vol. 106, n 1, p. 43-55.

4. Voir sur l'UOIF, Samir Amghar, «L'Union des Organisations Islamiques de France : la gestion politique de l'Islam ", à paraître, Cahiers Internationaux de Sociologie.

5. Farhad Khosrokhavar, L'islam des jeunes, Paris, Flammarion, 1997 (cf. Arch. 102.35). 
6. Claire de Galembert, « La gestion publique de l'islam en France et en Allemagne. De l'improvisation de pratiques in situ à l'amorce d'un processus de régulation nationale ", $L a$ Revue Internationale et Stratégique, $\mathrm{n}^{\circ}$ 52, hiver 2003-2004.

7. Voir Hervé Terrel, « L'État et la création du Conseil Français du Culte Musulman », in L'Islam en France, Cités, Paris, PUF, 2004.

8. Ces cas sont rares mais leurs effets sont percutants.

9. Voir par exemple Dounia Bouzar, «L'islam entre mythe et religion, Les nouveaux discours religieux dans les associations socioculturelles musulmanes ", Les Cahiers de la sécurité intérieure, 54, $4^{\mathrm{e}}$ trimestre 2003, p. 173-189.

10. Dès 1996, date de sa nomination, Jean-Pierre Chevènement lance certaines propositions audacieuses, déjà évoquées dans le rapport Marchand de 1990 : déroger provisoirement à l'article 2 de la loi de 1905 qui interdit le subventionnement direct et public des cultes, dans le cas de l'islam, pour réaliser un principe d'équité entre les religions.

11. L'émission du dimanche matin «Islam » est consacrée au processus de consultation les 12 et 19 mars 2000.

12. Voir rapport du « Comité synthèse et rédaction », créé le 31 mai 2000, dans un texte qui date du 12 juillet 2000 .

13. Al Istichâra, $\mathrm{n}^{\circ} 1$, mars $2000, \mathrm{p} .3$.

14. Voir l'éditorial d'Al Istichâra, $\mathrm{n}^{\circ} 1$, mars 2000.

15. Al Istichâra, $\mathrm{n}^{\circ} 1$, mars 2000, p. 6.

16. Al Istichâra, mars 2000 , p. 7.

17. Discours de Nicolas Sarkozy à la Grande Mosquée de Paris le 5 octobre 2002.

18. Nicolas Sarkozy, La république, les religions, l'espérance, Paris, Cerf, 2004, p. 87.

19. Nicolas SARKOZY, op. cit., p. 127-128.

20. Cf. Al Istichâra $\mathrm{n}^{\circ} 3$, page 3 .

21. Cf. Istichâra, no 3, p. 6-8.

22. Lieux de culte et lieux de sépulture ; structures associatives ; ministres du culte ; aumônerie ; Aïd el-Kebir ; pèlerinage ; audiovisuel.

23. Voir l'entretien à Libération du 21 février 2003.

24. Discours de Nicolas Sarkozy au rassemblement du Bourget de l'UOIF le 19 avril 2003, cité dans Le Monde du 22 avril 2003, p. 6.

25. Nicolas Sarkozy, La République, les religions, l'espérance, op. cit., p. 76-77.

26. Sur la discussion des chiffres, voir L'Islam en France, Cités, op. cit.

27. Interview de Nicolas Sarkozy, Libération du 21 février 2003. Je souligne.

28. Ce « modèle colonial » n'a pas forcément besoin de grandes institutions pour fonctionner. Comme le montrent Rémy Leveau et Catherine Wihtol de Wenden, il fonctionne aussi à travers les formes associatives locales qui ne peuvent échapper à « une approche en terme de sécurité et de lutte contre la déviance sociale ", in Rémy Leveau et Catherine Wihtol de Wenden, La Beurgeoisie. Les trois âges de la vie associative issue de l'immigration, Paris, CNRS éditions, 2001, p. 123.

29. Interview de Nicolas Sarkozy à Libération du 21 février 2003.

30. Voir aussi l'intervention de N. Sarkozy, in Libération du mardi 11 février 2003.

31. Cette pétition parait le 12 mai 2003 dans Le Figaro, et le 15 mai 2003 dans l'hebdomadaire Marianne.

32. Le Monde, 21 mai 2003, p. 11.

33. Voir la circulaire du président du CFCM aux CRCM, datée du 5 juillet 2004. 


\section{RÉSUMÉS}

Le CFCM qui voit le jour en avril 2003 est le résultat d'une volonté de reconnaissance politique de l'islam français, stratégie engagée par les gouvernements successifs depuis la fin des années 1980. Ce processus de reconnaissance politique est double : reconnaissance de la présence de l'islam par la République et acceptation de la part des représentants du culte des lois de la République, en particulier du principe de la laïcité. Cette reconnaissance réciproque donne lieu à des débats importants sur la place de l'islam dans la société française et sur sa visibilité dans l'espace public. Le décalage entre la mise en place d'une reconnaissance politique ponctuelle et l'absence de reconnaissance juridique due au principe de laïcité, peut être la source de tensions futures.

The creation of the Conseil Français du Culte Musulman, in April 2003, is the result of a strategy led by French state elites since the end of the 1980s. This political recognition is meant to give to the French Republic institutional interlocutors in the field of Islam as well as to have French Muslim elites recognize the laws and spirit of French secularism. This double process of recognition has produced political tensions in the public and political spaces: the disjunction between political recognition and the lack of legal recognition may lead in the future to more political tensions in the French public space.

El CFCM, creado en abril de 2003, es el resultado de una voluntad de reconocimiento político del Islam francés, estrategia elegida por los gobiernos sucesivos desde fines de los años '80. Este proceso de reconocimiento político es doble : reconocimiento de la república de la presencia del Islam, y aceptación por parte de los representantes del culto de las leyes de la República, en particular del principio de la laicidad. Este reconocimiento recíproco da lugar a debates importantes sobre el lugar del Islam en la sociedad francesa, y sobre la visibilidad en el espacio público. La dislocación entre la puesta en práctica de un reconocimiento político puntual y la ausencia de reconocimiento jurídico debido al principio de laicidad puede ser la fuente de tensiones futuras.

\section{AUTEUR}

MALIKA ZEGHAL

Centre d'Études Interdisciplinaires des Faits Religieux-CNRS 